$|\mathbf{C}| \mathbf{O}|\mathbf{P}|$ JM13714

\title{
WHAT IS THE SENSE IN LOGIC AND PHILOSOPHY OF LANGUAGE? ${ }^{1}$
}

\begin{abstract}
In the paper, various notions of the logical semiotic sense of linguistic expressions - namely, syntactic and semantic, intensional and extensional - are considered and formalised on the basis of a formal-logical conception of any language $L$ characterised categorially in the spirit of certain Husserl's ideas of pure grammar, Leśniewski-Ajdukiewicz's theory of syntactic/semantic categories and, in accordance with Frege's ontological canons, Bocheński's and some of Suszko's ideas of language adequacy of expressions of $L$. The adequacy ensures their unambiguous syntactic and semantic senses and mutual, syntactic and semantic correspondence guaranteed by the acceptance of a postulate of categorial compatibility of syntactic and semantic (extensional and intensional) categories of expressions of $L$. This postulate defines the unification of these three logical senses. There are three principles of compositionality which follow from this postulate: one syntactic and two semantic ones already known to Frege. They are treated as conditions of homomorphism of partial algebra of $L$ into algebraic models of $L$ : syntactic, intensional and extensional. In the paper, they are applied to some expressions with quantifiers. Language adequacy connected with the logical senses described in the logical conception of language $L$ is, obviously, an idealisation. The syntactic and semantic unambiguity of its expressions is not, of course, a feature of natural languages, but every syntactically and semantically ambiguous expression of such languages may be treated as a schema representing all of its interpretations that are unambiguous expressions.
\end{abstract}

\footnotetext{
${ }^{1}$ The paper was delivered at the conference ,,Formal Methods and Science in Philosophy III" at Inter-University Centre, Dubrovnik, Croatia, April 11-13, 2019. It is based on results presented in the author's papers [65, 66].
} 
Keywords: Logic and philosophy of language, categorial language, syntactic and semantic senses, intensional semantics, meaning, extensional semantics, denotation, categorisation, syntactic and semantic compatibility, algebraic models, truth, structural compatibility, compositionality, language communication.

\section{Introduction}

The word 'sense' has many meanings, and it appeals to us in many ways. On the basis of philosophy (and/or theology), it is for centuries that we have been trying to grasp and understand what the sense of our life is; likewise the sense of existence, the sense of our action and endeavour, and what the sense of the world is in general. From the point of view of philosophy, there are various visions and many theories regarding the sense of the world, the sense of life, our actions, etc. To discover their rational justifications, logical knowledge is needed, but, obviously, it is not enough. This philosophical meaning of the word 'sense' must clearly be distinguished from the logical, semiotic one. In the philosophical meaning, the word 'sense' is used as a certain property of extra-linguistic objects when it is said that something has or does not have sense, while referring to such objects. It derives from the basic, logical and semiotic meaning of this word, the meaning referring to linguistic objects, verbal signs. It should be noted, however, that it is not only the non-semiotic, but also the semiotic usage of the word 'sense' that is homogenous. Thus, one can speak of many notions of sense.

In this paper, we would like to characterise and formalise various notions of the logical and philosophical sense of linguistic expressions; from the viewpoint of logic, only these notions of sense can be of interest to us. The contemporary logic, logic of language (logical semiotics) can define the semiotic sense, logical sense strictly with regards to some general aspects of developing the cognition of the world and, at the same time, contributing to an explication of one of the most important traditional philosophical problems: Language adequacy of our knowledge in relation to cognition of reality, or, briefly: language adequacy. It is connected with the mutual relations between the three elements of the triad, reality-knowledge-language, and an adequate reflection of fragments of reality via expressions of language and inter-subjective knowledge of these fragments [67]. 
The above-mentioned adequacy requires, first of all, syntactic and semantic characterisation of language expressions as generalised by a grammar $[57,66]$. Languages structured by grammar and logic are important tools of thinking, cognition of reality and knowledge acquisition, which stand for the foundations of our sense of existence [43]. In modern logic and philosophy of language, an approach based on Frege functions. It is implemented by the trend of formal and logical reflection on language and Fregean senses.

Logical sense, in its different variants, is considered and formalised on the basis of the conception of formalisation of language $L$, which is sketched below. The syntactic sense of these expressions is defined on the basis of language syntax and semantic senses - on the basis of bi-level language semantic: intensional and extensional.

From the logical point of view, the three notions of the sense of expressions of language $L$ are understood as follows [62, 68] (see Fig. 1):

- syntactic sense is found in expressions of $L$ which are well-formed; it is their essence; it is defined in the syntax of $L$, and-in accordance with Carnap's distinction, intension-extension [21], or Frege's differentiation, Sinn-Bedeutung [23]-two kinds of semantic sense:

- intensional sense is proper to the expressions of $L$ which have a meaning, intension; it is defined in intensional semantics of $L$,

- extensional sense is proper to the expressions of $L$ which have a denotation, extension; it is defined in extensional semantics of $L$.

The syntactic and semantic notions of sense must be differentiated and explicated. This is possible through a conceptualisation of these notions that will lead to a formal-logical theory of syntax and semantics of language $L$, which specifies and describes these notions.

There are different points of view on the grammar of language, its syntax and semantics. In the paper, any language $L$, its syntax and bilevel semantic: intensional and extensional, is characterised and formalised categorially in the spirit of some ideas of Husserl (see [30]) and LeśniewskiAjdukiewicz's theory of syntactic/semantic categories [3, 4, 36, 37], in accordance with Frege's ontological canons [21], Bocheński's motto, syntax mirrors ontology [13], and some ideas of Suszko: language should be a linguistic scheme of ontological reality and simultaneously a tool of its cognition $[50,51,52,53]$. 


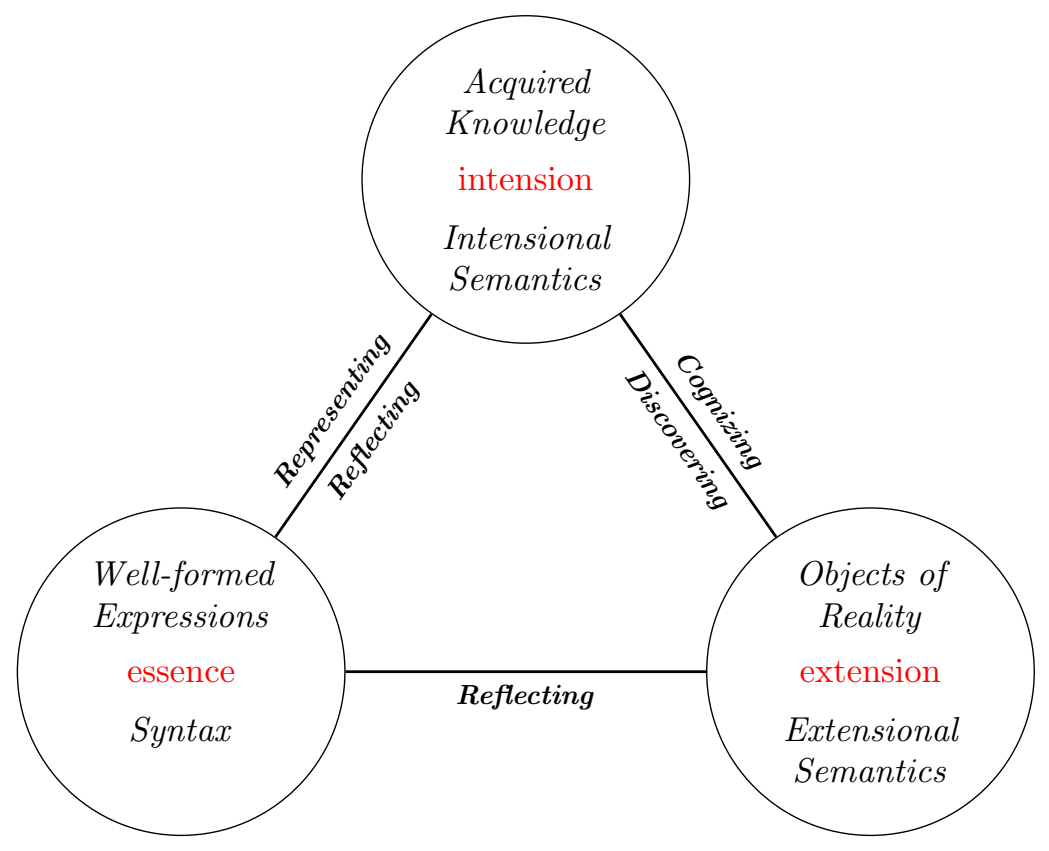

Fig. 1. Three notions of linguistic sense: essence, intension, extension.

\section{Main ideas of the formalisation of categorial language $L$}

Categorial language $L$ is defined if the set $S$ of all well-formed expressions (wfes) of $L$ is determined. These expressions must satisfy the requirements of categorial syntax and categorial semantics. The categorial syntax is connected with generating the set $S$ by the classical categorial grammar, the idea of which originated from Ajdukiewicz [3, 4] under the influence of Leśniewski's theory of semantic (syntactic) categories in his systems of protothetics and ontology [36, 37], under Husserl's ideas of pure grammar (see [30]), and under the influence of Russel's theory of logical types. The notion of categorial grammar was shaped by Bar-Hillel (see $[6,7,8]$ ) and developed by Lambek, Montague, Cresswell, Buszkowski, Marciszewski, Simons, Tałasiewicz and others $[14,15,16,17,18,19,20,21,34,35,38$, $39,40,45,46,52]$. The first formalisation of languages generated by the 
aforementioned classical categorial grammar, the notion introduced and explicated by Buszkowski was presented in the author's book in Polish [55] and its English translation, as well as some extension [56] (see also [63]).

In the categorial approach to language $L$, wfes of $S$ should belong to appropriate syntactic categories. A characteristic feature of categorial syntax is that each composed $w f e$ of the set $S$ has a functor-argument structure, so that it is possible to distinguish in it the main part (the so-called main functor) and the other parts (called arguments of this functor), yet each constituent of the wfe has a determined syntactic category. Categorial intensional and extensional semantics is connected with meaning and denotation of wfes of $S$ and with their membership in appropriate semantic categories: intensional and extensional, respectively (see [25, 61, 63, 64, 65, 66]). Each constituent of the composed wfe has a determined semantic (intensional and extensional) category, can have a meaning (intension) assigned to it, and thus also a category of knowledge (the category of constituents of knowledge) and also denotation (extension), and thus - an ontological category (the category of ontological objects).

The meanings (intensions) of wfes of $L$ are treated as certain constituents of inter-subjective knowledge: logical concepts, logical judgments, operations on such notions or judgments, on the former and the latter, on other operations.

Object references (references) of wfes of $L$, and also constituents of knowledge, are objects of the cognised reality: individuals (concretes or abstract), states of things, operation on the indicated objects, and the like. Denotations (extensions) of wfes of $L$ and constituents of knowledge are sets of such objects. The compatibility of these denotations is called semantic compatibility of $L$.

\section{General assumption concerning the logical sense of expressions of language $L$}

In the logical conception of language $L$ and the semiotic senses outlined in the paper, expressions of $L$ have syntactic, intensional and extensional senses and satisfy some general conditions of the logical sense of these expressions. Baseline conditions apply to syntactic and semantic unambiguity expressions of language $L$ and the subsequent - relate to categorial compatibility and structural compatibility. 


\subsection{Syntactic and semantic unambiguity}

The starting point is the syntactic and semantic unambiguity of the language expressions of language $L$. They should be:

- syntactically coherent and wfes of the set $S$ (its essences),

- structurally unambiguous: have one syntactic sense (essence), i.e. do not contain amphiboly and have the one mentioned functor-argument structure,

- semantically unambiguous: have one intension and one extension, thus, one meaning and one denotation.

Remark 1. Syntactic and semantic unambiguity is not, of course, a feature of natural languages and not often of languages of non-exact sciences, but every syntactically and semantically ambiguous expression of these languages may be treated as a schema representing all of its interpretations that are unambiguous expressions (with exactly one syntactic and/or semantic sense) and which serve for an adequate description of specified fragments of reality.

For example, the sentence:

Teachers are tired because they teach students in various schools and they have a lot of them.

is structurally ambiguous (contains amphiboly), but it can be treated as a schema of two unambiguous sentences:

Teachers are tired because they teach students in various schools and they have a lot of students.

and

Teachers are tired because they teach students in various schools and they have a lot of schools.

On the other hand, the structurally unambiguous sentences

I came back tomorrow on foot on the colourful black-and-white train of 25:66.

She laughed with sweet tears which fell weightlessly onto the ceiling.

have no meaning or intensional and extensional sense; they are semantic nonsense. 
In the categorial approach to language $L$, generated by the classical categorial grammar, a categorial index (type) $i(e)$ of a certain set $T$ of types is unambiguously assigned to every wfe $e$ of the set $S$, and every composed wfe of $S$ has the functor-argument structure. Categorial indices (types) were introduced into logical semiotics by Ajdukiewicz [3] with the goal of determining the syntactic role of expressions and to examine their syntactic connection, in compliance with the principle of syntactic connection (Sc) which, in a free formulation, says that:

(Sc) The categorial type of the main functor of each functor-argument expression of language $L$ is formed out of the categorial type of the expression which the functor forms together with its arguments, as well as out of the subsequent types' arguments of this functor.

Every functor-argument expression $e$ of $L$ can be written in a functionalargument form as follows:

$$
e=f\left(e_{1}, e_{2}, \ldots, e_{n}\right),
$$

where $f$ is the main functor of $e$ and $e_{1}, e_{2}, \ldots, e_{n}$ are its subsequent arguments. Then, assuming that $t$ is the type of $e$ and $t_{1}, t_{2}, \ldots, t_{n}$ are successive types of its arguments, the type of the functor $f$ satisfying the principle (Sc) can be written in the following quasi-fractional form:

$$
i(f)=i(e) / i\left(e_{1}\right) i\left(e_{2}\right) \ldots i\left(e_{n}\right)=t / t_{1} t_{2} \ldots t_{n} .
$$

Then, the set $S$ of all wfes of $L$ is defined as the smallest set including the vocabulary $V$ of $L$ and closed under the principle $(S c)$ :

DEFINITION 1.

$$
\begin{aligned}
& S=\bigcap\left\{X: V \subset X \wedge \forall e=f\left(e_{1}, e_{2}, \ldots, e_{n}\right)(S c(e)) \rightarrow e \in X\right\} \text { where, } \\
& S c(e)=\left(i(e)=t \wedge \forall j=1,2, \ldots n i\left(e_{j}\right)=t_{j}\right) \rightarrow i(f)=t / t_{1} t_{2} \ldots t_{n} .
\end{aligned}
$$

In the formal definition of set $S$, it is required that each functorargument constituent of the given expression should satisfy the principle (Sc).

Every wfe $e$ of $S$ is a meaningful expression of $L$ possessing one intension, i.e. one meaning $\mu(e)$, where $\mu$ is the operation of indicating the meaning defined on the set $S$ :

$$
\mu: S \rightarrow \mu(S)=K .
$$


The meaning $\mu(e)$ of the wfe $e$ of the set $S$ may be intuitively understood, in accordance with the understanding of meaning of expressions by Ajdukiewicz [1, 2] and, independently, by Wittgenstein [55] as a common property of all the wfes of $S$ which possess the same manner of using as does $e$ by competent users of language $L$ (cf. [41]). Formalisation of thus conceived notion of meaning (and related notions) is given by WybraniecSkardowska in [62]. In [62], its different philosophical conceptions, in particular those originating from Richard Montague, Donald Davison or Michael Dummett, are sketched. In my approach to the meaning of an expression of $L$, it is treated as a constituent of knowledge $K=\mu(S)$.

Every wfe e of $S$ is a meaningful expression of $L$ possessing one denotation, extension $\delta(e)$, where $\delta$ is the operation of denoting defined on set $S$ :

$$
\delta: S \rightarrow \delta(S)=O .
$$

The notion of denoting can, however, be introduced also as the operation of denoting $\delta_{K}$, defined on the set of constituents of knowledge $K$ :

$$
\delta_{K}: K \rightarrow \delta_{K}(K) \subseteq O .
$$

The denotation $\delta(e)$ of the meaningful expression $e$ is defined as the set of all ontological objects (or the ontological object) of the set $O=\delta(S)$, whose occurrences the expression $e$ refers to. The denotation $\delta_{K}(k)$ of the constituent $k$ of knowledge $K$ is defined as the set of all extra linguistic, ontological objects to which $k$ refers. Semantic compatibility takes place iff $\delta(S)=\delta_{K}(K)=O$ (see Fig. 2).

\subsection{Categorial compatibility}

In the logical conception of language $L$, the three distinguished kinds of logical sense of expressions of $L$ must be compatible: any wfe of $L$ having the syntactic sense, essence (belonging to a syntactic category of the defined kind), has a semantic, intensional sense (intension) and an extensional sense (extension) and is, simultaneously, a meaningful expression of $L$ belonging to a defined intensional and, respectively, to a defined extensional semantic category. The logical sense of wfes of $L$ is connected with the compatibility of their syntactic and semantic, intensional and extensional categories. In the categorial approach to language, the aforementioned categories of wfes of $L$ are determined by attributing to them, as to their 


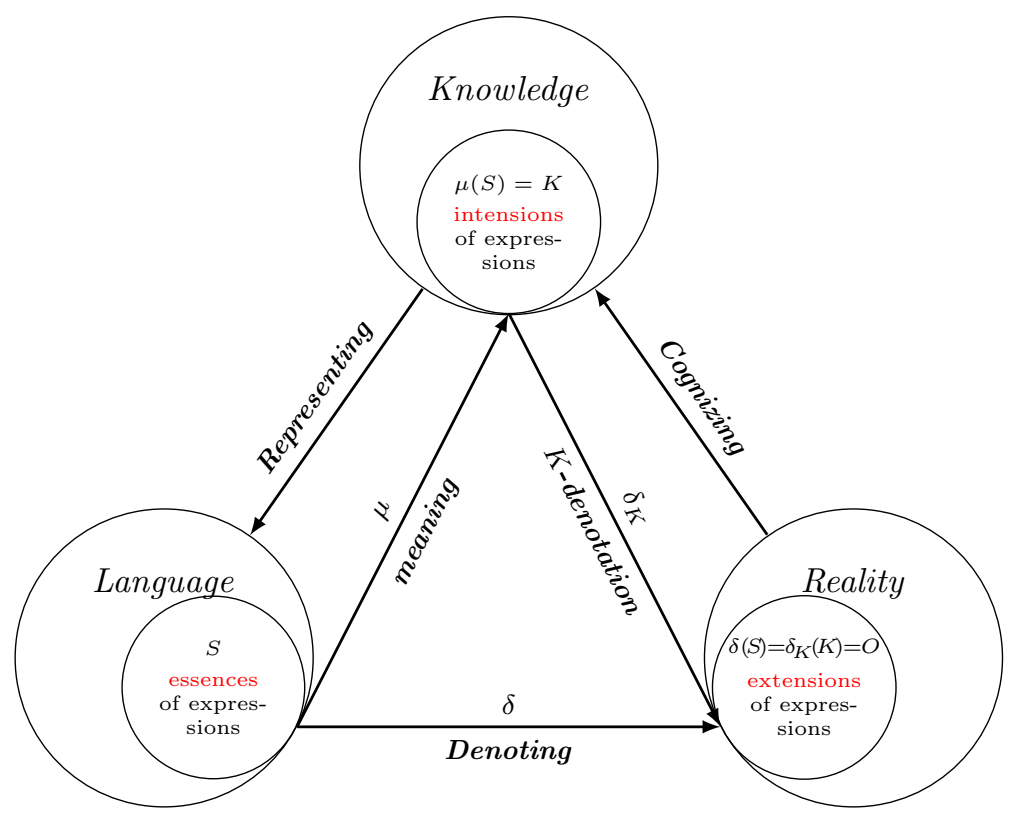

Fig. 2. Semantic compatibility and language adequacy.

expressions, categorial indices (types) of the set $T$. Compatible categories have the same categorial type that unifies these three notions of sense (see Fig. 3).

Categorial types play here the role of a tool coordinating meaningful expressions and extralinguistic objects: intensions and extensions $[4,47$, $50,51,52]$.

\subsubsection{Postulate of categorial compatibility}

The postulate of categorial compatibility of syntactic and semantic categories is one of the most important conditions of the logical sense of wfes of language $L$. Here is a more formal description of this postulate. Let

1. $S$ be the set of all wfes of $L$,

2. $K$ - the set of all intensions of expressions of the set $S ; K=\mu(S)$,

3. $O$ - the set of all extensions of expressions of the set $S$; $O=\delta(S)=\delta_{K}(K)$. 


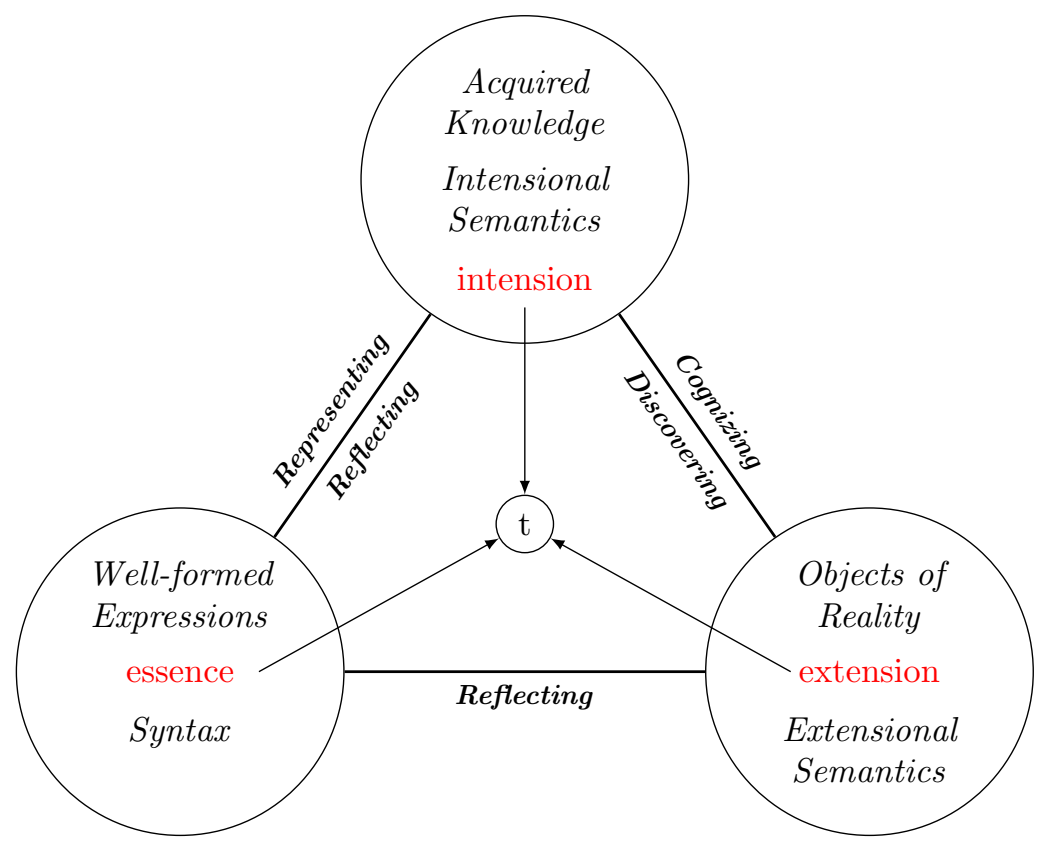

Fig. 3. Type-unifying three notions of logical sense: essence, intension, extension.

The above-discussed syntactic and semantic categories of meaningful wfes of $L$ are the following subsets of the set $S$ :

Definition 2. Syn $_{\boldsymbol{t}}=\{\boldsymbol{e} \in S: \boldsymbol{i}(\boldsymbol{e})=t\}, \quad$ where $\boldsymbol{i}: S \rightarrow T$,

Definition 3. Int $_{\boldsymbol{t}}=\left\{\boldsymbol{e} \in S: \boldsymbol{i}_{\boldsymbol{K}}(\mu(\boldsymbol{e})\}=t\right\}$, where $\boldsymbol{i}_{\boldsymbol{K}}: K \rightarrow T$,

Definition 4. $E k s_{\boldsymbol{t}}=\left\{\boldsymbol{e} \in S: \boldsymbol{i}_{\boldsymbol{O}}(\delta(\boldsymbol{e})\}=t\right\}$, where $\boldsymbol{i}_{\boldsymbol{O}}: O \rightarrow T$,

The syntactic (resp. intensional, resp. extensional) category with the index $\mathrm{t}$ is the set of all wfes of $S$ that have the categorial index $\mathrm{t}$ (resp. intensions of which, resp. extensions of which have the index t).

The postulate of categorial compatibility defining an aspect of the logical sense of wfes of $L$ has the following form [65, 66, 67]:

$$
\text { Syn }_{\boldsymbol{t}}=\text { Int }_{\boldsymbol{t}}=E k s_{\boldsymbol{t}} \quad \text { for any } t \in T .
$$




\subsubsection{Type-unifying logical senses}

The formal postulate $(\mathrm{P})$ does not grasp the problem of the logical sense of language expressions of $L$ adequately, because it does not show the relationships of the distinguished categories of wfes (essences) with the corresponding extra-linguistic categories of intensions and ontological categories of extensions in such a way that the mutual correspondence of elements of the triad: reality-knowledge-language, and the language adequacy of syntax with bi-level semantics, intensional and extensional, have been preserved (see Fig. 2).

As it was mentioned, unambiguous determined meanings (intensions) and denotations (extensions) should be assigned to wfes of $L$. They belong, respectively, to suitable extra linguistic categories of objects: categories of meanings, intensions (e.g. logical notions, logical judgments, operations on them) and ontological categories of denotations, extensions (e.g. individuals, set of individuals, states of affairs, or operations on them).

The categories of meanings, intensions, are subsets of the set $K$ of constituents of knowledge, and ontological categories - subsets of the set $O$ ontological objects. They are determined by categorial indices (types). And so, for any type $t \in T$ :

DeFinition 5. $K_{t}=\left\{m \in K: i_{K}(m)=t\right\}$,

Definition 6. $O_{t}=\left\{o \in O: i_{O}(o)=t\right\}$.

Sematic categories (see Definitions 3 and 4 ) can by defined by formulas: Corollary 1. Int $_{t}=\left\{e \in S: \mu(e) \in K_{t}\right\}$,

Corollary 2. Ext $_{t}=\left\{e \in S: \delta(e) \in O_{t}\right\}$,

stating that the semantic intensional (resp. extensional) category with the index $t$ is the set of all wfes of $L$, the meanings, intensions (resp. denotations, extensions) of which belong to the category of constituents of knowledge (resp. to the ontological category) with the type $t$.

It is easy to prove that for any $e \in S$ and $t \in T$, by Corollaries 1 and 2 we can state that the Axiom $(\mathrm{P})$ of categorial compatibility can be replaced by the following equivalent conditions:

TheOrem 1. $e \in$ Syn $_{t}$ iff $\mu(e) \in K_{t}$ iff $\delta(e) \in O_{t}$,

THEOREM 2. $i(e)=i_{K}(\mu(e))=i_{O}(\delta(e))$. 
So, we see that categorial types serve also as a tool coordinating wfes of $L$ and corresponding extra-linguistic objects, and that they unify the three notions of logical sense (see Fig. 4).

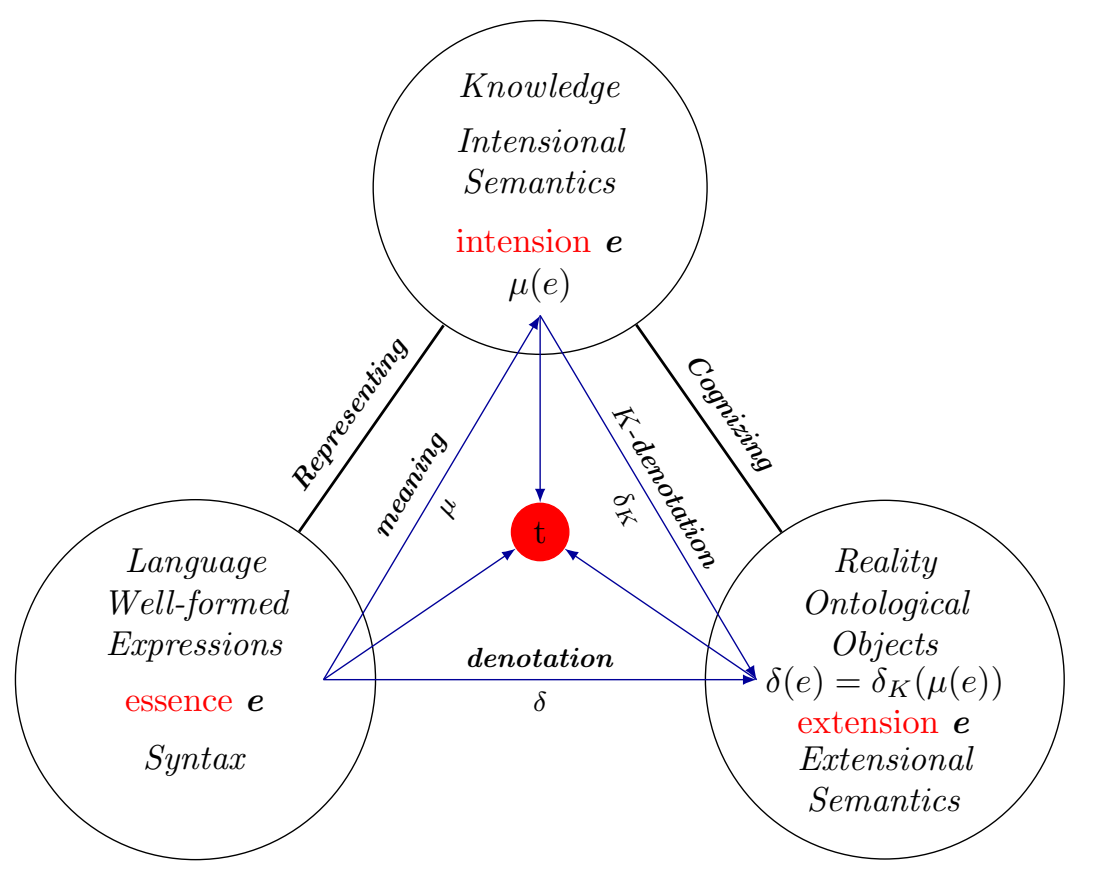

Fig. 4. Semantic compatibility and type - unifying the three notions of sense.

The idea of unification of the type of the logical term of the natural language, its intension and extension, is also one of the features of the type-theoretic object theory of E. Zalta [70, 71].

\subsubsection{Semantic compatibility}

From Diagrams 2 and 4, we conclude that ontological objects of the set $O$ are not only denotations of wfes of the set $S$ (its essences), but also denotations of intensions of knowledge $K$ corresponding to them. 
Semantic compatibility for language $L$ is defined by the following formula:

Definition 7. $\delta(e)=\delta_{K}(\mu(e) \in O$, where $\delta_{K}$ is the operation on intensions, meanings of knowledge $K$.

From Definition 7, it immediately follows that if two expressions of $L$ have the same meaning, then they have the same denotation:

Corollary 3. $\mu(e)=\mu\left(e^{\prime}\right) \rightarrow \delta(e)=\delta\left(e^{\prime}\right)$.

It is well known that the reverse implication does not hold. For example, the extensions of the terms 'equilateral triangle' and 'equiangular triangle' are the same, but their intensions are not.

\subsection{Structural compatibility}

\subsubsection{On the structure of expressions and their semantic counterparts}

The form of language expressions, their connectivity, well-formedness and logical sense, are connected with the structure of our knowledge and the structure of the cognising part of reality. Its language description is composed of parts that can be separated. Some of them are independent or relatively independent and are counted as basic language categories. In categorial languages, these are names and sentences. Others are auxiliary, dependent constituents of language expressions, which allow for the construction of more composed expressions from simpler ones. They are functors.

The categorial approach to $L$ allows us to define the structural compatibility of its composed expressions and their corresponding meanings and denotations. Every wfe of $L$ has one functor-argument structure. Functors of such expressions may be treated as partial functions defined on a proper subset of the set $S$ and with the values in this set. Language $L$ can then be characterised as the following partial algebra:

$\boldsymbol{L}=\langle S, F\rangle$, where $F \subset S$, and $F$ is the set of all functors of $L$.

As we mentioned in Sec. 3.1, (e), every composed expression $e$ of the set $S$ can be written in the functional-argument form:

$$
e=f\left(e_{1}, e_{2}, \ldots, e_{n}\right),
$$

where $f$ is the main functor and $e_{1}, e_{2}, \ldots, e_{n}$ its subsequent arguments. 
If the expression $e$ is $w f e$ of the set $S$, then - in accordance with the principle of syntactic connection (Sc) - the index of its main functor $f$, formed from the type $t$ of $e$ and successive types $t_{1}, t_{2}, \ldots, t_{n}$ of successive arguments $e_{1}, e_{2}, \ldots, e_{n}$ of the functor $f$, can be written in the following quasi-fractional form $(i(f))$ :

$$
i(f)=t / t_{1} t_{2} \ldots t_{n} .
$$

The functor-function $f$ corresponds to the function defined on meanings (intensions), respectively denotations (extensions) of arguments of this functor with subsequent types $t_{1}, t_{2}, \ldots, t_{n}$, the value of which is the intension, respectively the extension, of the expression $e$, which the functor $f$ forms, with the type $t$.

If, in language $L$, we have two basic syntactic categories, names and sentences with respective types $n$ and $s$, then meanings, intensions - logical notions with the type $n$ - are assigned to names, and meanings, intensions - logical judgments with the type $s$ - are assigned to sentences. Denotations of names are usually individuals or their sets, and denotations of sentences (in situational semantics) are states of affairs, situations. They also have, respectively, indices $n$ and $s$.

EXAMPLE. Let us consider the following sentence of a natural language:

$$
\text { Robert practices football. }
$$

with the index $s$, the main functor of which is the word 'practices' of two name arguments, 'Robert' and 'football', with the index $n$. The expression (i) can be written in the following function-argument form:

$$
\text { practices(Robert, football). }
$$

The index of the functor 'practices' is $s / n n$. The meaning of the functor (with the same index) is the function which, being defined on the notions of 'Robert' and 'football' with the index $n$, as meanings (intensions) of these names in the sentence (ii), has, as the value, its meaning, i.e. the logical judgment with the index s stating that Robert practices football. Denotation of the functor is the mapping which, being defined on denotations (denotates) of names in (ii) with index $n$, so on person Robert and the sport discipline football, has, as its value, the state of affairs: the fact that Robert practices football, being the denotation of the sentence (ii); it has, like the sentence, the index $s$. 
If somebody accepts, in accordance with Chomsky's phrase-structural grammar, that in (i) the main functor is 'practices football' (the predicate) of one argument 'Robert', then the function-argument form of (i) is as follows:

$$
\text { practices football }(\text { Robert })=(\text { practices }(\text { football }))(\text { Robert })
$$

The index of the composed functor 'practices football' is $s / n$, and the index of the functor 'practices' in it is $(s / n) / n$. Then the meaning and the denotation of the latter functor differ essentially from those used in (ii).

Remark 2. As we can see in a natural language, sentences may have a different functor-argument structure, thus different semantic senses: intensions and extensions. Therefore, they can be treated as skeletons, schemas which represent unambiguous expressions with one functor-argument structure, one meaning and one denotation.

\subsubsection{Principles of compositionality}

From the axiom $(\mathrm{P})$ of categorial compatibility three principles of compositionality follow $[63,64,65,66]$ : one syntactic (compositionality of essences, syntactic forms) and two semantic: compositionality of meaning (intension) and compositionality of denotation (extension). For every composed expression of $L$, the form $e=f\left(e_{1}, e_{2}, \ldots, e_{n}\right)$ and functions $h=i, \mu, \delta$, their common schema has the form:

$$
h(e)=h\left(\left(f\left(e_{1}, e_{2}, \ldots, e_{n}\right)\right)=h(f)\left(h\left(e_{1}\right), h\left(e_{2}\right), \ldots, h\left(e_{n}\right)\right) . \quad\left(C O M P_{h}\right)\right.
$$

For $h=i$, we have the syntactic principle, for $h=\mu, \delta$ we obtain the semantic principles corresponding to the ones already known to Frege [23] (cf. also [25, 31, 32, 39, 40, 42, 43, 26, 27, 28, 33]).

Speaking freely, these principles state that:

The categorial type (the syntactic form), resp. the meaning, resp. the denotation of a well-formed functor-argument expression of language $L$ is the value of the function of the type, resp. the function of the meaning, resp. the function of the denotation, of its main functor defined on types, resp. on meanings, resp. on denotations subsequent arguments of this functor. 


\subsubsection{Main properties of functions $h(f)$}

The formulation of the principle $\left(C O M P_{h}\right)$ defines $h(f)$ as functions. Indeed, index $i(f)$ of functor $f$ is the function:

$$
i(f):\left\{\left(i\left(e_{1}\right)\right\} \times\left\{i\left(e_{2}\right)\right\} \times \ldots \times\left\{i\left(e_{n}\right)\right)\right\} \rightarrow\{i(e)\},
$$

which, defined on $n$-tuple indices $\left(i\left(e_{1}\right), i\left(e_{2}\right), \ldots, i\left(e_{n}\right)\right)$, has the value $i(e)$; hence there follows the syntactic principle of compositionality $\left(C O M P_{i}\right)$.

Similarly, the meaning and the denotation of the functor $f$, defined on meanings and, respectively, on denotations of its arguments, are functions whose values are, respectively, meanings and denotations of the expression $e$. However, let us remember that the same $n$ argument functor $(n \geq 1)$, e.g. 'practices' in (ii) of Example 3.3.1, may have different arguments, though its meaning, respectively denotation, is uniquely determined.

Thus, for any wfe $e=f\left(e_{1}, e_{2}, \ldots, e_{n}\right)$ such that for types $i(e)=$ $t, i\left(e_{k}\right)=t_{k}$, where $k=1, \ldots, n, \mu(f)$, is the function:

$$
\mu(f): K_{t 1} \times K_{t 2} \times \ldots \times K_{t n} \rightarrow K_{t},
$$

which for intensions of arguments of functor $f$ has the value $\mu(e)$ compatible with the principle $\left(C O M P_{\mu}\right)$, and $\delta(f)$ is the function:

$$
\delta(f): O_{t 1} \times O_{t 2} \times \ldots \times O_{t n} \rightarrow O_{t},
$$

which for denotations of arguments of functor $f$ has the value $\delta(e)$ compatible with the principle $\left(C O M P_{\delta}\right)$.

Remark 3. Note that the logical sense of language expressions, including functors, assumes that they have both intensions and extensions. Thus, any functor $f$ forming the complex expression $e$ has the meaning $\mu(f)$ and at the same time denotation (reference) $\delta(f)$, and its meaning and denotation are functions that meet the conditions listed above in accordance with the semantic principles of compatibility.

In semiotic literature, however, we encounter some controversy regarding the sense of functors, which are predicates of name arguments in natural language sentences. Debate on Geach-Dummet controversy about the sense of a predicate is reconstructed by M. Tałasiewicz [53]. For Peter T. Geach sense of a predicate is its meaning and a function satisfying the principle of compositionality of meaning, while for Michael Dummet the sense is rather something that determines its denotation (reference). Tałasiewicz in [53] 
proposed a solution giving predicates both semantic senses as functions fulfilling the relevant conditions of semantic compositionality principles. This solution is interesting because it allows us to maintain semantic compatibility (see Def. 7).

\subsubsection{Generalisation of Ajdukiewicz's cancellation principles}

Just like the index of functor $f$ in expression $e=f\left(e_{1}, e_{2}, \ldots, e_{n}\right)(\operatorname{see}(i(f))$ in Sec. 3.1), we write its meaning and denotation in a quasi-fractional form. The general quasi-fractional form of the functions $h(f)$, for $h=i, \mu, \delta$ is given as the schema:

$$
h(f)=h(e) / h\left(e_{1}\right) h\left(e_{2}\right) \ldots h\left(e_{n}\right) .
$$

At the established quasi-fractional records $(\boldsymbol{i}(f))$ : the type of the functor $f,(\mu(f))$ of its meaning (intension) and $(\delta(f))$ of its denotation (extension), some counterparts of Ajdukiewicz's rules of cancellation of fractional indices (types) that serve to check the syntactic connection of complex expressions, correspond to the principles of compositionality $\left(C O M P_{h}\right)$. They follow from them. To justify these rules, it is sufficient to use the equality $\left(C O M P_{h}\right)$ from the left to the right and $(h(f))$. They allow us to calculate types, meanings (intensions) and denotations (extensions) of functor-argument expressions of $L$. Their schema, for $h=i, \mu, \delta$, can be written in the following way:

$$
h(e) / h\left(e_{1}\right) h\left(e_{2}\right) \ldots h\left(e_{n}\right)\left(h\left(e_{1}\right), h\left(e_{2}\right), \ldots, h\left(e_{n}\right)\right)=h(e) .
$$

EXAMPLE. For the functor 'practices' in the functor-argument sentence

$$
\text { practices(Robert, football) }
$$

the cancellation principles for $h=i, \mu, \delta$ have the forms:

$$
\begin{aligned}
& s / n n(n, n)=s, \\
& \mu((i i)) / \mu(\text { Robert } \mu(\text { football })(\mu(\text { Robert }), \mu(\text { football }))=\mu((\text { ii })), \\
& \delta((i i)) / \delta(\text { Robert }) \delta(\text { football })(\delta(\text { Robert }, \delta(\text { football }))=\delta((\text { ii })),
\end{aligned}
$$

while for the functor 'practices football' in the sentence

$$
\text { practices football }(\text { Robert })=(\operatorname{practices}(\text { football }))(\text { Robert })
$$

the cancellation principles for $h=i, \mu$ are the following: 
$((s / n) / / n(n))(n)=s / n(n)=s$,

$\mu($ practices football $($ Robert $))=((\mu($ practices $($ football $))(\mu($ Robert $))=$

$=(\mu($ practices $)(\mu($ football $))(\mu($ Robert $))=$

$=(((\mu($ iii $) / \mu($ Robert $)) / / \mu($ football $)(\mu($ football $))(\mu($ Robert $))=$

$=((\mu($ iii $) / \mu($ Robert $))(\mu($ Robert $))=\mu($ iii $)$.

Similarly, for $h=\delta$.

Let us observe that sentences (ii) and (iii) have the same categorial type $s$, and, according to Theorem 2, their intensions and extensions also have the type $s$. However, the appropriate constituents of these sentences and their intensions and extensions have different categorial types.

\subsubsection{Models of $L$ and the notion of truth}

The principles of compositionality can be considered as some conditions of homomorphisms $h=i, \mu, \delta$ of the syntactic algebra of language $\boldsymbol{L}$ into algebras of its images $h(\boldsymbol{L})$, i.e.

$$
\boldsymbol{L}=\langle S, F\rangle \underset{h}{\longrightarrow} h(\boldsymbol{L})\langle h(S), h(F)\rangle,
$$

where $F$ is the set of all simple functor-partial functions mapping subsets of set $S$ into set $S$, and $h(F)$, for $h=i, \mu, \delta$, is the set of functions corresponding to the functor-functions of set $F$.

Let us notice that the algebraisation of language can already be found in Leibnitz's papers. We can also find the algebraic approach to issues connected with syntax, semantics and compositionality in Montague's Universal Grammar [39] and in papers of Dutch logicians, especially in those by J. van Benthem $[9,10,11,12]$ and T.M.V. Jansen [31, 32]. The difference between their approaches and the approach which is presented here lies in fact that carriers of the syntactic and semantic algebras include functors, or, respectively, their suitable correlates, i.e. their $i$ - or semantic-function $\mu$ - and $\delta$ - images; simple functors and their suitable $i-, \mu-, \delta$ - images are simultaneously partial operations of this algebras. They are set-theoretical functions, determining those operations.

The algebra $i(L)=\langle i(S), i(F)\rangle$ is called the syntactic model of language $L$, while the algebras

$$
\mu(L)=\langle\mu(S), \mu(F)\rangle=\langle K, \mu(F)\rangle \text { and } \delta(\boldsymbol{L})=\langle\delta(S), \delta(F)\rangle=\langle O, \delta(F)\rangle
$$


are the semantic models for $L$; the first is called the intensional model for $L$, the other one, the extensional model for $L$.

In the process of cognition of reality, we want the sentences of the language $L$, representing the knowledge acquired about it, to be the carriers of true information about cognised portion of reality; they should be true in the above-mentioned models of $L$. Language as a tool for describing reality must distinguish the category of sentences among its syntactic categories. True sentences have informative content and allow us to enrich our knowledge. If for $h=i, \mu, \delta$, it is the case that the sentence e of language $L$ is true in models $h(\boldsymbol{L})$, we may say that our cognition by means of the sentence $e$ is true.

The notions of truthfulness in appropriate models are introduced theoretically by means of three new primitive notions $T h$, satisfying for $h=i, \mu, \delta$ the schema of axioms:

$$
\emptyset \neq T h \subseteq h(S)
$$

$\operatorname{AXIOM}(T h)$

and are understood intuitively, respectively, as the singleton consisting of the index of true sentences, the set of all true logical judgments, the set composed of the states of affairs that take place (in situational semantics) or the singleton composed of the value of truth (in Frege's semantics).

For $h=i, \mu, \delta$, we assume that:

Definition 8. The sentence $e$ of language $L$ is true in the model $h(\boldsymbol{L})$ iff $h(e) \in T h$.

In particular, if $h=\delta$, then we may state that the sentence $e$ of $L$ is true in the extensional model iff its extension is the state of affairs that takes place (in situational semantics), or it is the value of truth (in Fregean semantics).

\subsubsection{Some remarks concerning the problem of categories of first-order quantifiers}

There is a well-known problem with determining syntactic and semantic categories, and therefore a problem with categorial types of quantifiers, and, in particular, of quantifiers of the first order language $L 1$ and types of their intensions and extensions. To solve this problem, we can apply the principles of compositionality and the cancellation rules. Some general findings relating to the solution to the problem of syntactic categories of quantifiers, their denotation or/and meaning are presented in the following 
papers: $[58,59,69,70]$. In this work, I will limit myself to dealing with this problem for the quantifier in the simple formulas of $L 1$.

EXAMPLE. Let us consider the quantifier expressions:

$$
\text { (1) } \quad \forall_{x} P(x) \quad \text { and } \quad(2) \quad \exists_{x} P(x) \text {, }
$$

in which $P$ is an established one-argument predicate treated as a oneargument functor-function, and the quantifiers $\forall$ and $\exists$ are treated as twoargument functors-functions defined on a variable standing next to them and a sentential function with a free variable bound by the given quantifier. The categorial type for $x$ is $n_{1}$, i.e. $i(x)=n_{1}$, the type for $P$ is $s_{1} / n_{1}$, i.e. $i(P)=s_{1} / n_{1}$, because we assume that the type for the sentential function $P(x)$ is $s_{1}$, since $i(P(x))=i(P)(i(x))=s_{1} / n_{1}\left(n_{1}\right)=s_{1}$. The type of quantifiers $\forall$ and $\exists$ is then: $s / n_{1} s_{1}$, i.e. $i(\forall)=i(\exists)=s / n_{1} s_{1}$. Using the principles of compositionality and cancellation, we can 'compute' the type of the expression (1) in its functor-argument form:

$$
\begin{aligned}
i(\forall(x, P(x)) & =i(\forall)(i(x), i(P(x)))=i(\forall)(i(x), i(P)(i(x))) \\
& =s / n_{1} s_{1}\left(n_{1}, s_{1} / n_{1}\left(n_{1}\right)\right)=s / n_{1} s_{1}\left(n_{1}, s_{1}\right)=s .
\end{aligned}
$$

In a similar way, we 'calculate' the index of the expression $(2)=\exists(x, P(x))$. Thus, expressions (1) and (2) are sentences.

We will now define the denotation of the discussed quantifiers in Fregean semantics. We assume that $\delta(x)=U$, where $U$ is the universe of individuals in an established model $\boldsymbol{M}_{L 1} ; \delta(P): U \rightarrow \delta(P(x))$, where $\delta(P(x))=\delta(P)(\delta(x))=\{u \in U: \delta(P(x / u))=1\}$ and $P(x / u)$ is a sentence which we get for replacing in the sentential function $P(x)$ its free variable $x$ by the name of the individual $u$, and 1 is the value truth. Then,

$$
\begin{gathered}
\delta\left(\forall_{x} P(x)\right)=\delta(\forall)\left(\delta(x), \delta(P(x))=\left\{\begin{array}{lll}
1 & \text { if } & \delta(x)=U=\delta(P(x)) \\
0 & \text { if } & \delta(x)=U \neq \delta(P(x))
\end{array}\right.\right. \\
\delta\left(\exists_{x} P(x)\right)=\delta(\exists)\left(\delta(x), \delta(P(x))=\left\{\begin{array}{lll}
1 & \text { if } & \delta(x) \cap \delta(P(x)) \neq \emptyset \\
0 & \text { if } & \delta(x) \cap \delta(P(x))=\emptyset .
\end{array}\right.\right.
\end{gathered}
$$

So, the denotation $\delta(\forall)$ (resp. $\delta(\exists)$ ) of the quantifier $\forall$ (resp. $\exists$ ) is the function which, for the universe $U$ and the denotation of the scope of the quantifier, has the truth value if $f$ the denotation of its scope is the universe (resp. the denotation of this scope has at least one individual of the universe). 
In a similar way, we define the meanings of the quantifiers $\forall$ and $\exists$ in (1) and (2).

EXAMPLE. It is obvious that the quantifiers $\forall$ and $\exists$ are typically ambiguous in logic, depending on a type. In other contexts, e.g., in the expressions

$$
\begin{array}{ll}
\forall_{x, y} R(x, y) & \text { and } \\
\forall_{x} R(x, y) & \text { and }
\end{array}
$$

(4) $\exists_{x, y} R(x, y)$ or

(6) $\exists_{y} R(x, y)$

they have other categorial types, intensions and extensions. Their categorial type in expressions (3) and (4) is $s / n_{1} n_{1} s_{2}$, where $s_{2}$ is the index of the sentential function of two individual variables, while in expressions (5) and (6) they have the type $s_{1} / n_{1} s_{2}$. The predicate-functor's $R$ categorial type is, of course, $s_{2} / n_{1} n_{1}$.

It is easy to check and 'compute' that exemplary expressions are syntactically connective, therefore wfes. The first of them, (3) and (4), are sentences, because they have the index $s$, while the others, (5) and (6), are sentential functions with one free variable, because they have the index $s_{1}$.

\section{Final remarks}

The logical sense of language expressions is, of course, a kind of idealisation. In the logical and categorial conception of language, the sense of its expressions, both syntactic and semantic, intensional and extensional, ensures their structural and semantic unambiguity and mutual syntactic and semantic compatibility.

A natural language, and often also the scientific variation, is a living creature, still developing. The degree of syntactic and semantic senses of its expressions changes, it can be narrower or higher, depending on its skilful precision. However, structural or semantically ambiguous expressions can always be split into expressions having unambiguous syntactic and semantic senses and be categorially analysed. Also, expressions that are imprecise or vague can be replaced by sets of sentences with precise meanings and denotations. Moreover, they can be considered separately with respect to their categorial structure, because only expressions with a high degree of logical sense, syntactical and semantical (intensional and extensional), get closer to the sense and may, after a proper justification, become theorems of a given discipline of knowledge and be a base for satisfactory interpersonal communication about our world. 
Acknowledgements The author wishes to thank the Reviewer of this paper for comments and suggestions. Several remarks that he made led to some additions or improvement in the text.

\section{References}

[1] K. Ajdukiewicz, O znaczeniu wyrażen (On the meaning of expressions), [in:] The Commemorative Book of Polish Philosophical Society in Lvov (1931), pp. 31-77.

[2] Ajdukiewicz, Sprache und Sinn, Erkenntnis, vol. IV (1934), pp. 100-138.

[3] K. Ajdukiewicz, Die syntaktische Konnexität, Studia Philosophica 1, Leopoli, vol. 1, (1935), pp. 1-27. (English translation: Syntactic Connection, [in:] S. McCall (ed.), Polish Logic 1920-1939, Clarendon Press, Oxford (1967), pp. 202-231).

[4] K. Ajdukiewicz, Zwiqzki sktadniowe między cztonami zdań oznajmujacych (Syntactical Relations Between Constituents of Declarative Sentences), Studia Filozoficzne, vol. 6(21) (1960), pp. 73-86. (First presented in English at the International Linguistic Symposium in Erfurt, September 27-October 2, 1958).

[5] K. Ajdukiewicz, Język i poznanie (Language and Cognition), vol. 1, PWN, Warsaw (1960).

[6] K. Ajdukiewicz, Pragmatic Logic, Synthese Library, vol. 62, Reidel, Dordrecht-Boston, and PWN, Warsaw (1975).

[7] Y. Bar-Hillel, On Syntactical Categories, Journal of Symbolic Logic, vol. 15 (1950), pp. 19-37.

[8] Y. Bar-Hillel, A Quasi-arithmetical Notation for Syntactic Description, Language, vol. 29 (1953), pp. 47-58.

[9] Y. Bar-Hillel, Language and Information. Selected Essays on Their Theory and Applications, Addison-Wesley, Reading, Mass. (1950).

[10] J. van Benthem, Universal Algebra and Model Theory. Two Excursions on the Border, Report ZW-7908, Department of Mathematics of Groningen University, Groningen (1980).

[11] J. van Benthem, Why Is Semantics What?, [in:] J. Groenendijk, T. Janssen and M. Stokhof (eds.), Formal Methods in the Study of Language, Mathematical Centre Tract Amsterdam, vol. 135 (1981), pp. 29-49. 
[12] J. van Benthem, The Logic of Semantics, [in:] F. Landman and F. Veltman (eds.), Varieties of Formal Semantics, GRASS series, vol. 3, Foris, Dordrecht (1984), pp. 55-80.

[13] J. van Benthem, Essays in Logical Semantics, Reidel, Dordrecht (1986).

[14] J. M. Bocheński, On the Syntactical Categories, New Scholasticism, vol. 23 (1949), pp. 257-280.

[15] W. Buszkowski, Three Theories of Categorial Grammar, [in:] W. Buszkowski, W. Marciszewski and J. van Benthem (eds.), Categorial Grammar, John Benjamins Publishing Company, AmsterdamPhiladelphia (1988), pp. 57-84.

[16] W. Buszkowski, Logiczne podstawy gramatyk kategorialnych Ajdukiewicza Lambeka (Logical Foundations of Ajdukiewicz's-Lambek's Categorial Grammars), Logika i jej zastosowania (Logic and Its Applications), PWN, Warsaw (1989).

[17] W. Buszkowski, O klasycznej gramatyce kategorialnej (On classical categorial grammar), [in:] J. Pelc (ed.), Znaczenie i prawda (Meaning and Truth), Rozprawy Semiotyczne, PWN, Warsaw (1994), pp. 203-220.

[18] W. Buszkowski, Type Logics in Grammar, [in:] V. F. Hendrics, J. Malinowski (eds.), Trends in Logic: 50 Years of Studia Logica, Kluwer Academic Publishers, Dordrecht (2003), pp. 321-366.

[19] W. Buszkowski, W. Marciszewski and J. van Benthem (eds.), Categorial Grammar, John Benjamins Publishing Company, AmsterdamPhiladelphia (1988).

[20] M. J. Cresswell, Logics and Languages, Methuen and Co., London (1973).

[21] M. J. Cresswell, Categorial Languages, Studia Logica, vol. 36 (1977), pp. 257-269.

[22] R. Carnap, Meaning and Necessity, University of Chicago Press, Chicago (1947).

[23] G. Frege, Begriffsschrift, eine der arithmetischen nachbildete Formelsprache des reinen Denkens, Halle (1879).

[24] G. Frege, Über Sinn und Bedeutung, Zeitschrift für Philosophie und pilosophishe Kritik, vol. 100 (1892), pp. 25-50. (English translation [in:] H. Feigel and W. Sellars (eds.) Readings in Philosophical Analysis, AppletonCentury-Crofts, New York (1947) and also [in:] B. Beaney (ed.) The Frege Reader, Blackwell, Oxford (1997), pp. 151-171.)

[25] G. Frege, Begriffsschrift und andere Ausätze (I. Angelelli, ed.), Wissenschaftliche Buchgesellschaft/G. Olms, Darmstadt-Hildesheim (1964). 
[26] L. T. F. Gamut, J. van Benthem, J. Groenendijk, D. de Jongh, M. Stokhoft and $\mathrm{H}$. Vercuyl, Logic, Language and Meaning, vol. I: Introduction to Logic, vol. II: Intensional Logic and Logical Grammar, The University of Chicago Press, Chicago-London (1991).

[27] W. Hodges, Compositional Semantics for Language of Imperfect Information, Logic Journal of the IGPL, vol. 5(4) (1996), pp. 539-563.

[28] W. Hodges, Compositionality Is Not the Problem, Logic and Logical Philosophy, vol. 6 (1998), pp. 7-33.

[29] W. Hodges, Formal Features of Compositionality, Journal of Logic, Language and Information, vol. 10 (2001), pp. 7-28.

[30] E. Husserl, Logische Untersuchungen, vol. I, Halle (1900), vol. II, Halle (1901).

[31] T. M. V. Janssen, Compositionality, [in:] J. van Benthem and A. ter Muelen (eds.), Handbook of Logic and Language, Elsevier Science, Amsterdam-Lausanne-New York (1996), pp. 417-473.

[32] T. M. V. Janssen, Frege, Contextuality and Compositionality, Journal of Logic, Language and Information, vol. 10 (2001), pp. 115-136.

[33] M. Kracht, Interpreted Languages and Compositionality, Springer, Dordrecht-Heildeberg-London-New York (2001).

[34] J. Lambek, The Mathematics of Sentence Structure, American Mathematical Monthly, vol. 65 (1958), pp. 154-170.

[35] J. Lambek, On the Calculus of Syntactic Types, [in:] R. Jakobson (ed.) Structure of Language and its Mathematical Aspects. Proceedings of Symposia in Applied Mathematics, vol. 12, AMS, Providence, Rhode Island (1961), pp. 166-178.

[36] S. Leśniewski, Grundzüge eines neuen Systems der Grundlagen der Mathematik, Fundamenta Mathematicae, vol. 14 (1929), pp. 1-81.

[37] S. Leśniewski, Über die Grundlagen der Ontologie, Comptes rendus des sèances dela Société des Sciences et des Lettres de Varsovie, Classe II, vol. 23 (1930), pp. 111-132. Reprinted [in:] J. Jadacki (ed.) Stanisław Leśniewski. Pisma zebrane, Gesmmelete Schriften, Tom 2 (Band 2), Towarzystwo Naukowe Warszawskie (Société des Sciences et des Lettres de Varsovie), Semper, Warszawa (2015), pp. 724-766.

[38] W. Marciszewski, A Chronicle of Categorial Grammar, [in:] W. Buszkowski, W. Marciszewski and J. van Benthem (eds.), Categorial Grammar, John Benjamins Publishing Company, Amsterdam-Philadelphia (1988), pp. 7-22.

[39] R. Montague, Universal Grammar, Theoria, vol. 36 (1970), pp. 373-398. 
[40] R. Montague, Formal Philosophy: Selected Papers of Richard Montague (ed. and introd. R.H. Thomason), Yale University Press, New Haven, Conn. (1974).

[41] J. Pelc, A Functional Approach to the Logical Semiotics of Natural Language, [in:] J. Pelc (ed. and introd.), Semiotics in Poland 1894-1969, Synthese Library, Studies in Epistemology, Logic and Methodology of Science, vol. 119, PWN/Reidel, Dortrecht-Boston (1979), pp. 342-375.

[42] B. H. Partee, Compositionality, [in:] F. Landman and F. Veltman (eds.), Varieties of formal semantics. Proceedings of the fourth Amsterdam Colloqium, Foris, Dordrecht (1984), pp. 281-311.

[43] F. J. Pelletier, Did Frege believe Frege's Principle, Journal of Logic, Language and Information, vol. 10 (2001), pp. 87-114.

[44] W. V. Quine, Logic as a Source of Syntactical Insight, Journal of Symbolic Logic, vol. 31(3) (1966), pp. 496-497.

[45] P. Simons, Combinators and Categorial Grammar, Notre Dame Journal of Formal Logic, vol. 30(2), (1988), pp. 241-261.

[46] P. Simons, Languages with Variable-binding Operators: Categorial Syntax and Combinatorial Semantics, [in:] J. Jadacki, J. Paśniczek (eds.), The Lvov-Warsaw School - the new generation (Poznań Studies in the Philosophy of Sciences and Humanities, vol. 89), Rodopi, Amsterdam-New York (2006), pp. 239-268.

[47] B. Stanosz and A. Nowaczyk, Logiczne Podstawy Języka (Logical Foundations of Language), Ossolineum, Wrocław-Warsaw (1976).

[48] R. Suszko, Syntactic Structure and Semantical Reference, Part I, Studia Logica, vol. 8 (1958), pp. 213-144.

[49] R. Suszko, Syntactic Structure and Semantical Reference, Part II, Studia Logica, vol. 9 (1960), pp. 63-93.

[50] R. Suszko, O kategoriach syntaktycznych i denotacjach wyrażeń w językach sformalizowanych (On syntactic categories and denotations of expressions in formalized languages), [in:] Rozprawy Logiczne (Logical dissertations in honour of Kazimierz Ajdukiewicz), PWN, Warsaw (1964), pp. 193-204.

[51] R. Suszko, Ontology in the Tractatus of L. Wittgenstein, Notre Dame Journal of Formal Logic, vol. 9 (1968), pp. 7-33.

[52] M. Tałasiewicz, Philosophy of Syntax: Foundational Topics, Springer, Dordrecht (2010).

[53] M. Tałasiewicz, The Sense of a Predicate, [in:] P. Stalmaszczyk (ed.) Philosophy of Logic of Predication, Peter Lang (2017), pp. 231-277. 
[54] L. Wittgenstein, Philosophical Investigations, Blackwell, Oxford (1953).

[55] U. Wybraniec-Skardowska, Teoria języków syntaktycznie kategorialnych (The Theory of Syntactically Categorial Languages), PWN, Wrocław-Warszawa (1985).

[56] U. Wybraniec-Skardowska, Theory of Language Syntax. Categorial Approach, Kluwer Academic Publishers, Nijhoff International Philosophy Series. Dordrecht-Boston-London (1991), DOI: https://doi.org/10.1007/ 978-0-585-26680-0.

[57] U. Wybraniec-Skardowska, Logical and Philosophical Ideas in Certain Approaches to Language, Synthese, vol. 116(2) (1998), pp. 231-277.

[58] U. Wybraniec-Skardowska, On Denotations of Quantifiers, [in:] M. Omyła (ed.), Logical Ideas of Roman Suszko, Faculty of Philosophy and Sociology of Warsaw University, Warsaw (2001), pp. 89-119.

[59] U. Wybraniec-Skardowska, Three Principles of Compositionality, Bulletin of Symbolic Logic, vol. 7(1) (2001), pp. 157-158.

[60] U. Wybraniec-Skardowska, O pojęciach sensu z perspektywy logiki (On the Notions of Sense from the Perspective of Logic), [in:] K. Trzęsicki (ed.) Ratione et Studio, Publishing House of Białystok University, Białystok (2005), pp. 155-190.

[61] U. Wybraniec-Skardowska, On the Formalization of Classical Categorial Grammar, [in:] J. Jadacki and J. Paśniczek (eds.) The Lvov-Warsaw School - the new generation (Poznań Studies in the Philosophy of Sciences and Humanities, vol. 89), Rodopi, Amsterdam-New York (2006), pp. 269-288.

[62] U. Wybraniec-Skardowska, Meaning and Interpretation, Studia Logica, vol. 85(1) (2007), pp. 105-132 (Part I), DOI: https://doi.org/10.1007/ s11225-007-9026-0; vol. 85(2) (2007), pp. 263-276 (Part II), DOI: https://doi.org/10.1007/s11225-007-9031-3.

[63] U. Wybraniec-Skardowska, On Metaknowledge and Truth, [in:] D. Makinson, J. Malinowski and H. Wansing (eds.), Trends in Logic: Towards Mathematical Philosophy, Springer, Berlin-Heidelberg (2009), pp. 319343, DOI: https://doi.org/10.1007\%2F978-1-4020-9084-4_16

[64] U. Wybraniec-Skardowska, Three Principles of Compositionality, [in:] U. Żegleń (ed.), Cognitive Science and Media in Education. From Formal and Cognitive Aspects of Language to Knowledge, no. 1, Publishing House Adam Marszałek, Torun (2010), pp. 28-65.

[65] U. Wybraniec-Skardowska, On Language Adequacy, Studies in Logic, Grammar and Rhetoric, vol. 40(53) (2015), pp. 257-292. 
[66] U. Wybraniec-Skardowska, Logic and Sense, Philosophy Study, vol. 6(9) (2016), pp. 554-568, DOI: https://doi.org/10.17265/2159-5313/2016.09.007.

[67] U. Wybraniec-Skardowska, Categories of First Order Quantifiers, The Bulletin of Symbolic Logic, vol. 22(3) (2016), pp. 427-428, DOI: https://doi.org/10.1017/bsl.2016.22

[68] U. Wybraniec-Skardowska, Categories of First Order Quantifiers, [in:] A. Garido, U. Wybraniec-Skardowska (eds.) The Lvov-Warsaw School. Past and Present, Springer-Birkhäuser, Cham, Switzerland (20168), pp. 763-777, DOI: https://doi.org/10.1007/978-3-319-65430-0_54

[69] U. Wybraniec-Skardowska, Logic and Ontology of Language, [in:] B. Skowron (ed.), Contemporary Polish Ontology, De Gruyter Mouton, Berlin (2019), pp. 109-132.

[70] E. N. Zalta, Abstract Objects: An Introduction to Axiomatic Metaphysics, Reidel, Dordrecht (1983).

[71] E. N. Zalta, Intensional Logic and the Metaphysics of Intentionality, MIT Press, Cambridge, MA (1988).

[72] E. N. Zalta, Typed Object Theory, [in:] Book of Abstracts of the Context, Cognition and Communication Conference, June 2018, University of Warsaw, Warsaw (2018), p. 189.

Cardinal Stefan Wyszyński University in Warsaw

Department of Philosophy

Warsaw, Poland

email: skardowska@gmail.com 